\title{
Coproporfiria hereditaria
}

\section{Hereditary coproporhyria}

\author{
Jorge Andrés Hurtado-Mayor, Juliana Castro-Henao, María Camila Salazar-Cuevas, \\ Freddy Andrés Barrios-Arroyave, Mónica Duque-Arias • Pereira (Colombia)
}

DOI: https://doi.org/10.36104/amc.2021.1950

\section{Resumen}

La coproporfiria hereditaria $(\mathrm{CPH})$ es un trastorno congénito, autosómico dominante, que se presenta en aproximadamente dos a cinco personas por cada millón de habitantes a nivel mundial. Constituye un reto diagnóstico en aquellos pacientes que cursan con dolor abdominal agudo. Se presenta el caso de una adolescente de 17 años que debutó con un cuadro atípico de dolor abdominal sin etiología clara. Se le documentó porfobilinógeno elevado en orina y fue tratada con hematina. El estudio genético realizado concluyó condición de portadora hererocigota de la variante nonsense patogénica c.717T>A; p.Cys239 en el gen CPOX, permitiendo diagnosticar una coproporfiria hereditaria. La literatura nacional disponible ha presentado casos con diagnóstico de porfiria aguda intermitente en pacientes con dolor abdominal de origen no específico, sin abordar las clasificaciones existentes de las porfirias hepáticas y sin detallar el diagnóstico genético, por lo cual el presente caso es un aporte a la casuística nacional. (Acta Med Colomb 2021; 46. DOI: https:// doi.org/10.36104/amc.2021.1950).

Palabras clave (DeCS): porfirias hepáticas, diagnóstico diferencial, dolor abdominal, pruebas genéticas, coproporfiria hereditaria.

\section{Abstract}

Hereditary coproporphyria (HCP) is a congenital, autosomal dominant disorder which occurs in approximately two to five people per million inhabitants, worldwide. It is a diagnostic challenge in patients with acute abdominal pain. We present the case of a 17-year-old adolescent who debuted with atypical abdominal pain with no clear etiology. Elevated urinary porphobilinogen was found, which was treated with hematin. A genetic study concluded that the adolescent was a heterozygous carrier of the c.717T>A; p.Cys239 pathogenic nonsense mutation in the CPOX gene, leading to a diagnosis of hereditary copropophyria. The available national literature has presented cases diagnosed with acute intermittent porphyria in patients with abdominal pain of unknown origin, without covering the existing classifications of hepatic porphyrias and without detailing the genetic diagnosis; thus, this case is a contribution to the national case studies. (Acta Med Colomb 2021; 46. DOI: https://doi.org/10.36104/amc.2021.1950).

Keywords: hepatic porphyrias, differential diagnosis, abdominal pain, genetic tests, hereditary coproporphyria.
Jorge Andrés Hurtado-Mayor, Juliana CastroHenao, María Camila Salazar-Cuevas: Estudiantes de Medicina, Institución Universitaria Autónoma de las Américas; Dr. Freddy Andrés Barrios-Arroyave: $\mathrm{PhD}(\mathrm{c})$ Epidemiología y Bioestadística. Docente Facultad de Medicina, Institución Universitaria Autónoma de las Américas; Dra. Mónica Duque-Arias: Especialista en Medicina Crítica y Cuidado Intensivo Pediátrico, Clínica los Rosales. Pereira (Colombia). Correspondencia: Dr. Freddy Andrés BarriosArroyave. Pereira (Colombia).

E- Mail: freddyandresbarrios@yahoo.com Recibido: 15/VII/2020 Aceptado: 6/IV/2021

\section{Introducción}

Las porfirias son un grupo de alteraciones de tipo metabólico secundarias a la deficiencia en enzimas encargadas de la biosíntesis del Hem, que se caracterizan por la acumulación de porfirinas y sus precursores tóxicos como el porfobilinógeno y ácido aminolevulínico (1). Se han descrito cinco tipos principales de porfirias hepáticas: porfiria aguda intermitente (PAI), porfiria variegada (PV), coproporfiria hereditaria $(\mathrm{CPH})$ y porfiria por deficiencia de ácido 5-aminolevulínico deshidratasa (ADP) (2-4).
La CPH es consecuencia de una alteración en la enzima coproporfirinógeno oxidasa, resultado de la mutación del gen $C P O X$, ubicado en la banda cromosómica $3 q 12$ (1). Los síntomas aparecen después de la pubertad e incluyen manifestaciones neuroviscerales debidas a la acumulación de precursores de porfirina tóxicos como dolor abdominal, acompañado de neuropatías agudas y signos inespecíficos: constipación, vómito, hipertensión, taquicardia, entre otras manifestaciones neurológicas y psiquiátricas, que no permiten establecer una etiología con facilidad (2, 5-7). La falta 
de reconocimiento clínico, la demora en el diagnóstico y el uso de recursos innecesarios genera retrasos en el tratamiento (8).

La importancia de este caso radica en su diagnóstico clínico y genético, lo cual no está descrito en los reportes de caso de la literatura nacional. El objetivo es contribuir a la casuística regional y nacional publicada y aportar elementos para la discusión actual sobre el abordaje y manejo de la $\mathrm{CPH}$.

\section{Presentación del caso}

Femenina de 17 años, universitaria, sin antecedentes de importancia, procedente de Pereira, con cuadro clínico de cuatro días de evolución caracterizado por dolor abdominal intenso, urente, de localización epigástrica que posteriormente migró a fosa ilíaca derecha, asociado a múltiples episodios eméticos e hiporexia, por lo cual consultó a urgencias. Al ingresar a la institución de tercer nivel de atención, se encontró a la exploración física un abdomen doloroso a la palpación en fosa iliaca derecha, signo de psoas positivo y punto de McBurney doloroso, por lo que realizan presunción diagnóstica de apendicitis aguda. Fue valorada por cirujano pediatra quien orientó el caso hacia una patología de origen urogenital, se solicitó uroanálisis y ecografía abdominal, con reporte de infección de vías urinarias y ultrasonografía sin alteraciones, descartando una condición quirúrgica. Se ordenó manejo antibiótico ambulatorio y egreso.

Dos días después, la paciente consultó nuevamente por exacerbación del dolor abdominal, sin respuesta a la terapia analgésica. Se dio manejo sintomático y se practicó un hemograma, sin alteraciones; uroanálisis sin hallazgos patológicos; gonadotropina coriónica subunidad beta cualitativa negativa en orina. Se hospitalizó e inició manejo con dipirona (40 mg/kg/día, vía intravenosa) y morfina ( $2 \mathrm{mg}$ / dosis vía intravenosa) sin lograr un control del dolor. Posteriormente se instauró tratamiento con sulfato de magnesio por hipomagnesemia reportada en laboratorio con valores de $1.2 \mathrm{mg} / \mathrm{dl}$.

La paciente refirió dolor abdomino-pélvico acompañado de disuria y se evidenció puño percusión renal bilateral positiva. La ecografía urinaria reportó dilatación pielocalicial de predominio derecha lo que orientó a etiología por uropatía obstructiva, pero con tomografía de vías urinarias negativa para dicha presunción diagnóstica.

Posteriormente, presentó episodios disociativos con cuadro de agitación psicomotora, asociado a hiponatremia $(111 \mathrm{mg} / \mathrm{dl})$ e hipocalemia $(2.28 \mathrm{mg} / \mathrm{dl})$, por lo que se sospechó una porfiria con síntomas neuropsiquiátricos. Las pruebas de Hoesch y Watson-Schwarz resultaron positivas, además de los niveles altos en orina de 24 horas de: ácido 5-aminolevulínico (12 mg/g, valor de referencia 0-5 mg/g) y porfobilinógeno $(18 \mathrm{mg} / 24$ horas, valor de referencia menos de $4 \mathrm{mg} / 24$ horas). Se solicitó haptoglobina y anticuerpos antinucleares, con valores dentro de rangos normales. Fue trasladada a terapia intensiva por hiponatremia severa sin- tomática. Se inició hematina a dosis de $4 \mathrm{mg} / \mathrm{kg} /$ día vía intravenosa, durante una semana con recuperación progresiva.

Se practicó estudio genético de análisis de secuenciación y deleciones-duplicaciones mediante NGS (Next Generation Sequencing) para genes de porfiria, analizando los genes: ALAD, ALAS2, C15ORF41, COPX, FECH, HFE, HMBS, PROX, SLC19A2, UROD, UROS, todos ellos evaluados con una cobertura de $100 \%$. Se concluyó que la paciente era portadora heterocigota de la variante nonsense c.717T >A; . Cys239 en el gen CPOX. Los resultados de este estudio molecular soportaron el diagnóstico de $\mathrm{CPH}$.

Posterior al egreso hospitalario la paciente continuó tratamiento con nutrición y hematología. No ha tenido nuevas crisis y su condición actual es estable.

\section{Discusión}

La PAI es la más frecuente de todas las porfirias, con 1.5 por cada 100000 personas en el mundo. Sin embargo, la CPH es aún más rara, se presenta entre uno a nueve casos por millón $(9,10)$.

La mutación del gen CPOX genera una disfunción mitocondrial en la enzima coproporfirinógeno oxidasa (2). La acumulación del porfobilinógeno y el ácido aminolevulínico son los responsables de las manifestaciones neuroviscerales y psiquiátricas (2), así como se presente en este caso, en el cual la paciente debutó con dolor abdominal agudo y posterior cuadro de agitación psicomotora acompañado de episodios disociativos.

La variedad de las manifestaciones clínicas hizo difícil su diagnóstico, por la evolución tórpida del cuadro clínico. Inicialmente no se encontró una etiología clara, solicitando paraclínicos que dieron lugar a diferentes presunciones diagnósticas sin éxito.

Identificar los factores precipitantes es útil para evitar los episodios agudos o exacerbaciones. Estos factores incluyen cambios hormonales como el aumento de la progesterona, al igual que el estrés metabólico y el consumo de medicamentos que incrementan la actividad hepática del citocromo P450, entre otros (5). Debido a que inicialmente no se tenía entre las consideraciones diagnósticas la porfiria, a la paciente se le suministró dipirona como analgésico (familia de las pirazolonas, asociado con episodios agudos y considerado inseguro en porfiria), siendo un exacerbante de las manifestaciones neuroviscerales, además de ser el desencadenante para la instauración de una encefalopatía hiponatrémica, aumentando el riesgo de mortalidad (11-13).

En la literatura nacional se describe la porfiria como un dilema diagnóstico, debido a su presentación clínica inespecífica (8), lo que es concordante con el caso clínico expuesto, en el que se tuvieron diversas presunciones diagnósticas, por lo que el tiempo para determinar su etiología se prolongó.

Los casos clínicos más recientemente publicados en Colombia hacen referencia a pacientes con PAI, como los descritos por Torregrosa (14), Erazo (15), Lozano (16) y Latorre (17). En países como Argentina, únicamente hemos 
encontrado publicado un reporte de caso clínico de $\mathrm{CPH}$ en una serie de casos de porfiria (18). Por lo tanto, se considera que la presentación de este caso es de utilidad para la comunidad médica, aportando al reconocimiento de esta entidad y recordando que existe una variedad de porfirias hepáticas que deben tenerse en cuenta antes de dar un diagnóstico definitivo. Los hallazgos de este caso y el abordaje genético difieren de los reportes presentados en dichas publicaciones.

La presencia de vacíos epidemiológicos en relación con la ocurrencia de CPH a nivel regional, puede explicarse posiblemente, por la determinación diagnóstica usual que es basada en cuantificación de niveles de PBG en orina y por el cuadro clínico, que hace que en la mayoría de las ocasiones se oriente el caso hacia una porfiria hepática tipo PAI, que es la más común, sin realizar una adecuada confirmación mediante estudio genético específico, respaldado esto por Jaramillo y cols., quienes describieron que $98 \%$ de las porfirias hepáticas de su análisis de 101 pacientes, se diagnosticaron como PAI sin tener el respaldo genético para diferenciar entre esta y la CPH o la PV (19).

Así, el diagnóstico genético molecular es fundamental para diferenciar a una $\mathrm{CPH}$ de otras porfirias. En esta paciente se identificó una variante nonsense heterocigota en el gen $C P O X$, que consiste en el cambio de una timina por una adenina en la posición $717 \mathrm{del} A D N c(c .717 T>A)$, que a nivel proteico genera modificación nonsense de una cisteína por un codón de parada prematuro (7). Esta variante no ha sido reportada en las bases de datos ClinVar (20) ni en HGMD (The Human Gene Mutation Database) (21), y genera una proteína truncada prematuramente, llevando posiblemente a una pérdida de función de esta o a una degradación del transcrito de ARNm por el mecanismo NMD (nonsense mediated decay) (22). Adicionalmente, la pérdida de función es un mecanismo patogénico conocido en este gen. Las mutaciones en el gen CPOX (OMIM*612732), localizado en la región cromosómica $3 q 11$.2, son causantes de la $\mathrm{CPH}$, entidad de herencia autosómica dominante (7).

Se identificaron divergencias entre este caso y las descripciones similares de CPH a nivel mundial: Insiripong y cols, reportaron un caso también en una persona joven (21 años), diferenciándose en que el nuestro no cursó con convulsiones, hipertensión, insuficiencia respiratoria ni falleció, además que en dicho reporte no se describe diagnóstico genético. En común se tiene que se halló orina de color rojo oscuro con prueba positiva de Watson-Schwartz y que tampoco se pudo identificar un factor precipitante claro, pese a que a nuestra paciente se le identificó y trató una infección urinaria (23).

Por otro lado, el presente caso se diferencia del publicado por Sadie y cols, quienes reportaron la enfermedad en un paciente de mayor edad (39 años) y con una combinación de síntomas gastrointestinales y manifestaciones neurocutáneas (24). En cuanto a la publicación de Graziadei y cols, se tiene en común la presencia de un diagnóstico genético, aunque su paciente fue identificada con una nueva variante de sentido erróneo en el gen coproporfirinógeno oxidasa, y la mutación $c .1348 A>G$ (p.Arg450Gly), que se segregó en otros tres miembros de la familia. Adicionalmente, su paciente no presentó alteraciones urinarias de PBG (25). A diferencia del paciente descrito por Lagos y cols, en nuestro caso no se documentaron afectaciones a nivel renal ni neurológico (26).

Por su parte, el manejo de la crisis aguda de esta paciente fue adecuado de acuerdo con la literatura, puesto que se administró hematina, líquidos dextrosados y sulfato de magnesio $(14,27)$.

Como punto pendiente por resolver se considera la ausencia de un estudio genético extendido a familiares en primer grado de consanguinidad para determinar patrones de transmisión de la enfermedad. En este sentido, el papel de las pruebas genéticas en las porfirias agudas autosómicas dominantes (PAI, CPH y PV) es identificar los portadores presintomáticos de la mutación patógena específica de la familia, para que puedan recibir asesoramiento sobre cómo minimizar su riesgo de sufrir un ataque agudo (28).

La ausencia de reconocimiento y el bajo índice de sospecha de la $\mathrm{CPH}$ entre sus diagnósticos diferenciales puede retrasar el diagnóstico y tratamiento. Asimismo, genera un incremento en los costos asociados a la prestación del servicio por larga estancia hospitalaria o terapia intensiva (16).

Debido a su baja frecuencia, la CPH se convierte en un reto para el médico tratante, quien debe considerar este diagnóstico como una posible causa de dolor abdominal agudo con una evaluación clínica que no sugiera otra etiología (29). Nuevas investigaciones que podrían arrojar luz sobre esta enfermedad en Colombia se sustentarían a partir de la revisión de casos de porfirias hepáticas agudas que ya han realizado autores como Jaramillo y cols (19), pero faltaría complementar la casuística nacional con diagnósticos genéticos que permitan establecer a futuro la prevalencia exacta de $\mathrm{CPH}$ y otras variedades en nuestro país.

\section{Referencias}

1. Lambie D, Florkowski C, Sies C, Raizis A, Siu WK, Towns C. A case of hereditary coproporphyria with posterior reversible encephalopathy and novel coproporphyrinogen oxidase gene mutation c.863T $>\mathrm{G}$ (p.Leu288Trp). Ann Clin Biochem. 2018;55:616-9.

2. Valle G, Carmine Guida C, Nasuto M, Totaro M,Aucella F, Frusciante V, et al. Cerebral Hypoperfusion in Hereditary Coproporphyria (HCP): A Single Photon Emission Computed Tomography (SPECT) Study. Endocr Metab Immune Disord Drug Targets. 2016;16:39-46.

3. Kadish K, Smith K, Guilard R. Handbook of Porphyrin Science with Applications to Chemistry, Physics, Materials Science, Engineering, Biology and Medicine. Singapore. World Scientific Publishing; 2014. 434 p.

4. Cacheux V, Martasek P, Fougerousse F, Delfau M, Druart L, Tachdjian G, et al. Localization of the human coproporphyrinogen oxidase gene to chromosome band 3q12. Hum Genet [Internet]. 1994 [citado 18 de abril de 2020];94: 557-9. Disponible en: http://link.springer.com/10.1007/BF00211026

5. Singal A, Anderson KE. Hereditary coproporphyria [Internet]. UpToDate. 2020 [citado 5 julio 2020]. Disponible en: https://www.uptodate.com/contents/ hereditary-coproporphyria

6. Grimes R, Gilleece Y, Appleby T, Stockwell S, Pinto-Sander N, Sahabandu T, et al. A case of hereditary coproporphyria precipitated by efavirenz. AIDS. 2016;30:2142-3.

7. Wang B, Bissell DM. Hereditary Coproporphyria. 2012 Dec 13 [Updated 2018 Nov 8]. In: Adam MP, Ardinger HH, Pagon RA, et al., editors. GeneReviews ${ }^{\circledR}$ [Internet]. Seattle (WA): University of Washington, Seattle; 1993-2020. Disponible 
en: https://www.ncbi.nlm.nih.gov/books/NBK114807/

8. Montoya M, Barranco LA, Herrera F. Porfiria aguda. Dilema diagnóstico y manifestaciones neuroendocrinas. Acta Med Colomb. 2017;42:140-3.

9. DEYBACH J-C. Acute intermittent porphyria [Internet]. Orphanet. 2009 [citado 14 mayo 2020]. Disponible en: https://www.orpha.net/consor/cgi-bin/OC_Exp. php?lng $=$ EN \&Expert $=79276$

10. DEYBACH J-C. Hereditary coproporphyria [Internet]. Orphanet. 2009 [citado 14 mayo 2020]. Disponible en: https://www.orpha.net/consor/cgi-bin/OC_Exp. php?lng=EN\&Expert $=79273$

11. Norwegian Porphyria Centre (NAPOS). The drug database for acute porphyria [Internet]. Metamizole (dipyrone). 2018 [citado 10 de julio de 2020]. Disponible en: http://www.drugs-porphyria.org/monograph2.php?id=3546

12. Nikolova I, Petkova V, Tencheva J, Benbasat N, Voinikov J, Danchev N. Metamizole: A Review Profile of a Well-Known "Forgotten" Drug. Part II: Clinical Profile. Biotechnol. Biotechnol. Equip.2013;27:3605-19.

13. Moore MR, McColl KEL, Goldberg A. Drugs and the acute porphyrias. Trends Pharmacol. Sci. 1981;2:330-4

14. Torregrosa L, Ayala JC, Cervera S, Villa CA. La porfiria aguda intermitente y el cirujano: un reto diagnóstico Reporte de un caso y revisión de la literatura. Rev. Colomb. Cir. 2011;26:131-7.

15. Erazo-Narváez AF, Erazo MS, Zamora-Bastidas TO. Porfiria intermitente aguda como desencadenante de síndrome de Guillain-Barre. Rev. Fac. Med. 2019;67:349-55

16. Lozano A, Tovar OJ, Ortiz CA. Porfiria aguda: reporte de caso y revisión de tema. Rev. Fac. Med. 2008;16:106-14.

17. Latorre S, Castro J, Mendoza F. Neuropatía periférica como forma de presentación inicial en ataque agudo de porfiria. Reporte de caso clínico. Repert. Med Cir. 2017;26:39-43.
18. Melito VA, Rossetti MV, Parera VE, Batlle A. Porfirias poco frecuentes. Casos detectados en la población argentina. Rev Argent Dermatol 2006;87: 1-6.

19. Jaramillo-Calle DA, Aguirre Acevedo DC. Acute Hepatic Porphyrias in Colombia: An Analysis of 101 Patients. JIMD Rep. 2018; 44: 65-72.

20. ClinVar [Internet]. ClinGen Clinical Genome Resource. 2018 [citado 10 junio 2020]. Disponible en: https://www.clinicalgenome.org/data-sharing/clinvar/

21. The Human Gene Mutation Database [Internet]. HGMD®. 2017 [citado 10 junio 2020]. Disponible en: http://www.hgmd.cf.ac.uk/ac/index.php

22. Brogna S, Wen J. Nonsense-mediated mRNA decay (NMD) mechanisms. Nat Struct Mol Biol 2009;16:107-13.

23. Insiripong S, Tulayalak P, Ungpakorn R. Hereditary coproporphyria: a case report. J Med Assoc Thai 1993;76:53-55.

24. Sadie H, Stanfield M. Case Report of Hereditary Coproporphyria Accompanied by Nystagmus and Vestibular Dysfunction. J Med Cases. 2018;9:355-59.

25. Graziadei G, Spinelli D, Granata F, Brancaleoni V, Di Pierro E. Presentación inusual de hereditaria Coproporfiria: informe de un caso. Neurol Disord Stroke Int. 2018; 1: 1010

26. Lagos Maldonado A, Borrego García H, Ruiz Sancho A. Acerca de un caso de coproporfiria hereditaria con afectación neurológica, hematológica y renal. CARTA CIENTÍFICA. 2019; 42: 438-9.

27. Lecha M, Herrero C, Ozalla D. Diagnosis and treatment of the hepatic porphyrias. Dermatol Ther 2003;16:65-72.

28. Whatley SD, Badminton MN. Role of genetic testing in the management of patients with inherited porphyria and their families. Ann. Clin. Biochem. 2013;50:204-216.

29. Anderson KE, Bloomer JR, Bonkovsky HL, Kushner JP, Pierach CA, Pimstone NR, et al. Recommendations for the diagnosis and treatment of the acute porphyrias. Ann Intern Med 2005; 142: 439-450. 